\title{
Determination of the Lifetime in As Doped a-Se from Transient Photocurrents using Laplace Transform Technique
}

\author{
F. SERDOUK* AND M.L. BENKHEDIR \\ Laboratoire de Physique Appliquée et Théorique, LPAT, Université Larbi Tébessi-Tébessa, Algérie
}

\begin{abstract}
The identification, in our earlier papers, of the discrete energy levels in stabilized a-Se indicate that doping with As in the limit of $0.2-0.5 \%$ suppresses the shallow defect and increases the density of the deep one. It may be added at this point that an undesirable decrease in the hole lifetime $\tau$ was found to accompany the introduction of As in the a-Se lattice, which was attributed to a new hole trap. More analysis is clearly needed to resolve these questions. For these reasons we have developed a technique to calculate the lifetime. The obtained results confirm the image of the density of states near the valance band.
\end{abstract}

DOI: 10.12693/APhysPolA.134.168

PACS/topics: stabilized a-Se, transient photocurrent (TPC), multitraping model, high resolution Laplace transform method, density of localized states (DOS), lifetime $(\tau)$

\section{Introduction}

It is generally agreed that the distribution of density of states (DOS) in the upper half of the mobility gap near the conduction band mobility edge is not monotonous but contains peaks at certain energy levels [1-4]. The DOS distribution in the lower half of the mobility gap near the valence band edge is still surrounded by various uncertainties and controversies.

The density of states studied in detail by Kasap et al. $[5,6]$ is a featureless, monotonically decreasing distribution in energy. According to Benkhedir et al. [7], in pure a-Se above the valance band edge $E_{\mathrm{v}}$ DOS consists of a shallow defect level at $\sim 0.25 \mathrm{eV}$ above $E_{\mathrm{v}}$ and a defect level at $\sim 0.45 \mathrm{eV}$. The later was proposed earlier by Song et al. [1]. The shallow one is connected to a change in the dihedral angle while the deeper one is the $\mathrm{D}^{-}$center in the negative- $U$ model $[1,8]$. This image of the DOS is built, essentially, on the transient photoconductivity, TPC and TOF.

Later Serdouk and Benkhedir confirmed these results using the high resolution Laplace transform method [9]. A good agreement between the measured and the simulated currents was found. The key element in interpreting the transport properties in amorphous selenium is the determination of the carrier drift mobility and lifetime $\tau$. Several studies have discussed and demonstrated the scientific and technological importance of these parameters $[10,11]$.

The values of the drift mobility and lifetime in stabilized selenium were extracted from time of flight and interrupted-field time-of-flight experiments. The addition of low percentages of As makes it possible to im-

*corresponding author; e-mail: iserdouk@yahoo.fr prove several properties but causes large changes in the hole lifetime. It will be important to use the treatment of TPC traces using high resolution Laplace transform method to calculate the lifetime of holes in agreement with the found DOS above the valence band, and to compare the found results with experimental data, to check the validity of our proposed DOS model in the lower half of the mobility gap [9].

In the present paper we clarify this crucial issue. We focused our work on the determination of transport parameters. We show that the transient photoconductivity technique commonly used for determining the density of localized states can be used to obtain additional parameters, compared to its conventional use. Due to a new analysis, this technique allows us to examine the lifetime of holes in stabilized amorphous selenium.

\section{Method of simulation}

The calculations of the lifetime involve solving the continuity equation in the presence of deep trapping. About 20 years ago, Naito [12] was able to solve the system of equations governing the charge transport that describe multitraping model formulated by Noolandi [13], using high Laplace transform. The carrier rate equation is therefore expressed as:

$$
\begin{aligned}
& \frac{\mathrm{d} p(t)}{\mathrm{d} t}=-\sum_{i} \frac{\mathrm{d} p_{i}(t)}{\mathrm{d} t}-\frac{p(t)}{\tau}+n_{0} \delta(t), \\
& \frac{\mathrm{d} p_{i}(t)}{\mathrm{d} t}=\omega_{i} p(t)-\gamma_{i} p_{i}(t),
\end{aligned}
$$

where $p(t)$ is the instantaneous hole density, $p_{i}(t)$ is the density of holes occupying the traps, $\omega_{i}$ is the capture rate constant and $\gamma_{i}$ is the release rate constant. The expression obtained for the density of localized states is: 


$$
g(E)=\frac{I(0)}{\sigma v_{\mathrm{th}} k_{\mathrm{B}} T} \frac{\hat{I}(s) \hat{K}(s)-2[\hat{J}(s)]^{2}}{[\hat{I}(s)]^{3}} s^{2},
$$

with $J(t)=t I(t), H(t)=t^{2} I(t), s$ is the Laplace variable, $\sigma$ is the capture cross section and $v_{\text {th }}$ is the thermal velocity. The experimental and the theoretical Laplace transforms are defined through the following expressions:

$$
\begin{gathered}
I(s)=\sum_{i=0}^{N}\left[I\left(t_{j+1}\right) \mathrm{e}^{-s t_{j+1}}+I\left(t_{j}\right) \mathrm{e}^{-s t_{j}}\right] \\
\times \frac{\left[t_{j+1}-t_{j}\right]}{2}, \\
\hat{I}(s)=\frac{I(0)}{s+\frac{1}{\tau}+s \int_{0}^{E_{\mathrm{F}}} \frac{\sigma v_{\mathrm{th}} g(E)}{s+v \exp \left(-E / k_{\mathrm{B}} T\right)} \mathrm{d} E} .
\end{gathered}
$$

The calculation method proposed in this work follows the steps below:

- We start our analysis from a distribution of the localized states determined from the data of the transient photoconductivity, through the method based on the high Laplace transform.

- We calculate the Laplace transform of the experimental and theoretical transient photocurrents for each temperature using Eqs. (4) and (5), respectively.

For the numerical processing of the hole lifetime, we minimize the error between calculation and experiment. We make adjustments until a good fit using the least square method is obtained.

\section{Results and discussion}

Two types of structures are modeled. The final optimized DOS distribution near the valence band edge was found by Serdouk and Benkhedir, and the results are published in their paper [9]. The DOS distribution is given by:

$$
\begin{gathered}
g(E)_{0.2 \mathrm{at} \% \mathrm{As}}=6.636 \times 10^{21} \exp (-37.027 E) \\
+1.949 \times 10^{15} \exp \left[-\left(\frac{E-0.406}{0.046}\right)^{2}\right], \\
g(E)_{0.5 \mathrm{at} \% \mathrm{As}}=4.074 \times 10^{23} \exp (-28.785 E) \\
+1.593 \times 10^{18} \exp \left[-\left(\frac{E-0.375}{0.045}\right)^{2}\right] .
\end{gathered}
$$

In this section we apply the above method to the analysis of the results obtained by the experimental study of the hole transport in stabilized a-Se [14]. The fits listed in Fig. 1 are the "best" fits. The agreement between the experiment and the theory is satisfactory. The lifetime values for the a-Se samples doped with As are summarized in Table I. As seen in Table I, the hole lifetime is of the same order of magnitude and is unchanged in the two cases.

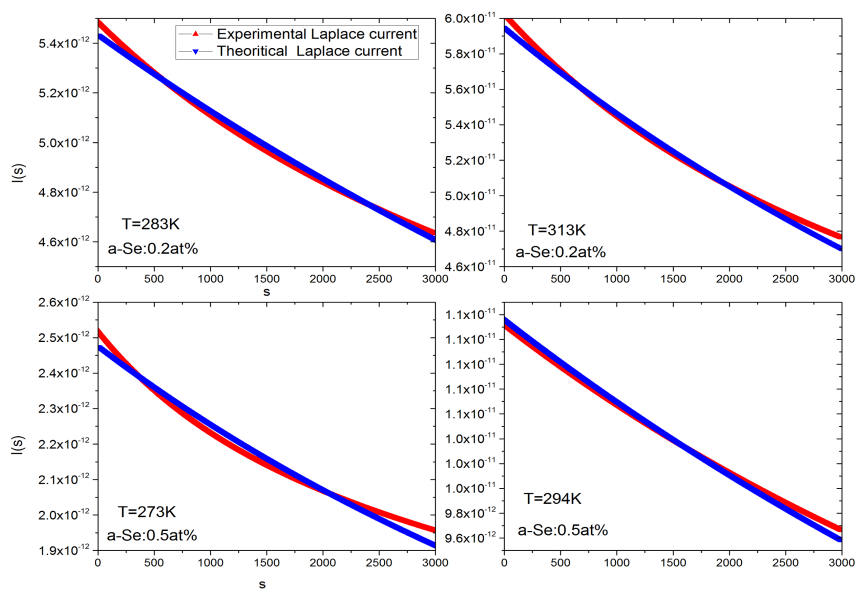

Fig. 1. Comparison of the Laplace transient photocurrent calculated using least square method in stabilized a-Se.

Alloying a-Se with small amounts of As decreases the hole lifetime. The results of transport measurements in pure a-Se [15] indicate that the values are in the range of $10-50 \mu \mathrm{s}$. It should be noted that the hole range decreases because the hole drift mobility is unaffected by addition of As, as reported in [16]. The decrease in the hole lifetime translates the changing of the populations. As mentioned in [17], deep trap concentration depends on the As concentration.

The density of states plotted in Figs. 3, 4 and 5 from [9], clearly shows that the concentration of deep hole traps increases with increasing arsenic content. Indeed, the carrier lifetime is inversely proportional to the concentration of deep traps. This interpretation is further supported by the approach adopted in [16]. We have also used the same method of simulation to investigate the effect of temperature on the hole lifetime. The numerical results are presented in Fig. 2.

It is clear that temperature influences the $\tau$ values. From $253 \mathrm{~K}$ to $313 \mathrm{~K}$ the hole lifetime decreases with increasing temperature, in full agreement with the pioneering study by Kasap et al. [18]. The temperature dependence of $\tau$ of the type reported for holes by Kasap et al. [18] indicates that the hole lifetime is thermally activated. In order to obtain the activation energy we use the Arrhenius equation. By plotting the logarithm of $\tau$ versus the corresponding inverse temperatures, a linear regression has been applied to the data of Table I, as shown in Fig. 2. The fitting parameters are presented in Table II.

The large value of the correlation coefficient indicates that the hole lifetime follows an exponential depen- 
dence. The slope of the line gives an activation energy of $E_{\tau \mathrm{h}}=(0.15114 \pm 0.1081) \mathrm{eV}$. Similar plots have been made which confirm the agreement between calculations and experimental values [18]. In the latter work, the hole activation energy is $E_{\tau \mathrm{h}}=0.18 \mathrm{eV}$.

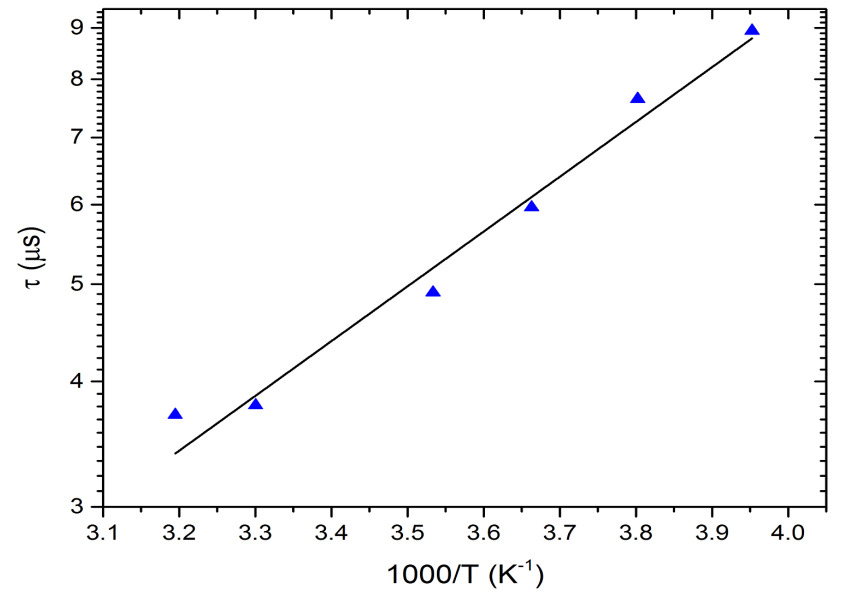

Fig. 2. Arrhenius plot for hole lifetime in stabilized a-Se.

Simulated hole lifetime in stabilized selenium

TABLE I

\begin{tabular}{c|c|c}
\hline \hline Temperature $[\mathrm{K}]$ & $\tau[\mu \mathrm{s}]$ & $\begin{array}{c}\text { Correlation } \\
\text { coefficient R }\end{array}$ \\
\hline 253 & 8.94 & 0.975 \\
263 & 7.645 & 0.972 \\
273 & 5.963 & 0.985 \\
283 & 4.905 & 0.994 \\
294 & 4.170 & 0.99 \\
303 & 3.787 & 0.994 \\
313 & 3.705 & 0.995
\end{tabular}

Various fitting parameters

TABLE II

\begin{tabular}{c|c|c}
\hline \hline Slope & Intercept & $\begin{array}{c}\text { Correlation } \\
\text { coefficient } R\end{array}$ \\
\hline $1256.72508 \pm 88.03145$ & $16.60962 \pm 0.31328$ & 0.97605
\end{tabular}

\section{Conclusions}

This study focuses on the determination of the lifetime. The found hole lifetime is around several micro seconds. It increases with decreasing temperature and it is not affected by the studied As concentrations. The results seem to fully satisfy all observed experimental characteristics and support our density of states model for As doped a-Se near the valance band that contains a feature at about $0.45 \mathrm{eV}$ above $E_{\mathrm{v}}$.

\section{References}

[1] H.Z. Song, G.J. Adriaenssens, E.V. Emelianova, V.I. Arkhipov, Phys. Rev. B 59, 10607 (1999).

[2] N. Qamhieh, M.L. Benkhedir, M. Brinza, G.J. Adriaenssens, J. Optoelectron. Adv. Mater. 7, 1781 (2005).

[3] C. Koughia, Z. Shakoor, S. Kasap, J. Marshall, J. Appl. Phys. 97, 033706 (2005).

[4] E. Emelianova, M. Benkhedir, M. Brinza, G. Adriaenssens, J. Appl. Phys. 99, 083702 (2006).

[5] S. Kasap, C. Koughia, J. Berashevich, R. Johanson, A. Reznik, J. Mater. Sci.: Mater. Electron. 26, 4644 (2015).

[6] C. Koughia, A. Reznik, C. Allen, R. Johanson, S. Kasap, Phys. Status Solidi A 213, 1856 (2016).

[7] M.L. Benkhedir, M. Brinza, G.J. Adriaenssens, C. Main, J. Phys.: Condens. Matter 20, 215202 (2008).

[8] M.L. Benkhedir, M.S. Aida, N. Qamhieh, A. Stesmansa, G.J. Adriaenssens, J. Optoelectron. Adv. Mater. 7, 329 (2005).

[9] F. Serdouk, M.L. Benkhedir, Physica B: Cond. Mat. 459, 122 (2015)

[10] S. Kasap, J. Phys. D: Appl. Phys. 33, 2853 (2000).

[11] M.Z. Kabir, S. Kasap, Appl. Phys. Lett. 80, 1664 (2002).

[12] N. Ogawa, T. Nagase, H. Naito, J. Non-Cryst. Solids 266-269, 367 (2000).

[13] J. Noolandi, Phys. Rev. B 16, 4466 (1977).

[14] M.L. Benkhedir, F. Djefaflia, M. Mansour, N. Qamhieh, Phys. Status Solidi C 7, 877 (2010).

[15] J.C. Shottmiler, M.D. Tabak, G. Lucovsky, A.T. Ward, J. Non-Cryst. Solids. 4, 80 (1970).

[16] S. Kasap, K. Koughia, B. Fogal, G. Belev, R. Johanson, Semiconductors 37, 789 (2003).

[17] S. Kasap, J. Rowlands, J. Mater. Sci.: Mater. Electron. 11, 179 (2000).

[18] B. Fogal, S. Kasap, Can. J. Phys. 92, 634 (2014). 